\title{
Senior-Loken syndrome. Case reports of two siblings and association with sensorineural deafness
}

\author{
Michael P Clarke, Timothy J Sullivan, Christopher Francis, Reuben Baumal, Theo Fenton, \\ William G Pearce
}

\begin{abstract}
Two siblings with Senior-Loken syndrome are described. The need for a full evaluation of renal function and hearing in children with a retinal dystrophy is emphasised.
\end{abstract}

Early onset rod cone dystrophies have been described in association with a number of renal lesions. The most common is that associated with nephronophthisis (medullary cystic disease). We report two siblings with the triad of nephronophthisis, retinal dystrophy, and sensorineural hearing loss.

\section{Case reports}

Two siblings (the older female, the younger male) were the products of a first cousin marriage between parents of Indian subcontinent origin.

CASE 1

The girl presented at 2 years of age with nystagmus. Vision was reduced in both eyes and the nystagmus was rapid, fine, horizontal, and pendular. There was a low myopic refractive error and the fundus was within normal limits. A diagnosis of autosomal recessive achromatopsia was made.

By the age of 9 years she complained of poor vision particularly in dim light. Her visual acuity was counting fingers at 7 feet $(2 \mathrm{~m})$ in the right eye and at 10 feet $(3 \mathrm{~m})$ in the left eye. Near vision was N36 in each eye. Colour vision was normal. Rapid, fine, horizontal pendular nystagmus was still present. Examination of the fundi showed narrowing of the retinal arterioles and pink optic discs with no pigmentation.

An electroretinogram (ERG) was performed at this time which showed no response. The diagnosis was revised to Leber's congenital amaurosis.

Audiological testing at this time demonstrated mild to moderate sensorineural hearing loss.

The following year she presented with fatigue, pallor, and nocturia. There was glycosuria and generalised aminoaciduria characteristic of a renal tubular defect. Her haemoglobin was $62 \mathrm{~g} / 1$ and her creatinine clearance $2.5 \mathrm{ml} / \mathrm{min} / 1.73 \mathrm{~m}$.

Renal ultrasound showed slightly shrunken kidneys and numerous cysts in the corticomedullary region. A renal biopsy showed severe chronic tubulointerstitial nephritis and global glomerulosclerosis affecting more than $90 \%$ of the glomeruli. Immunofluorescence was negative for immunoglobulin and complement.

On electron microscopy there was thickening of tubular basement membrane which was split into multiple layers. The glomerular basement membrane was normal.

The diagnosis of end stage renal failure secondary to nephronophthisis was made and peritoneal dialysis was started. Ophthalmic examination at this time showed a visual acuity of perception of light only in the right eye and 20/200 in the left eye. The nystagmus was now coarse and slow but remained pendular. The anterior segments were normal with no evidence of keratoconus or lenticonus. The refractive error was $-5 \cdot 50 \mathrm{DS} /+1 \cdot 25 \mathrm{DC} \times 090$ in both eyes. The optic discs were now pale and arterioles were attenuated. There was no evidence of retinal pigmentation or white spots in the fundus.

Her sensorineural hearing loss had become more profound, and she was fitted with bilateral hearing aids.

CASE 2

The brother of case 1 was seen at the age of 6 months when his sister first presented. He did not fixate or follow unless the target was large. There was rapid fine horizontal pendular nystagmus. There was a low hyperopic refractive error and the fundus was within normal limits.

Over the next few years global developmental delay became apparent and a seizure disorder developed. A computed tomographic (CT) head scan was normal.

An abdominal ultrasound at age 7.5 years showed his kidneys to be of normal size and to contain corticomedullary cysts. Renal biopsy was similar to his sister's but showed less advanced disease. He was found to have moderate sensorineural hearing loss. At $7 \cdot 5$ years his ophthalmic examination showed him to be able to follow a light and to have positive optokinetic nystagmus (OKN) in each eye. Nystagmus was fine, horizontal, and jerky. The anterior segments were normal with no keratoconus or lenticonus, and the refractive error was $-1.00 \mathrm{DS} /+2.00$ $\mathrm{DC} \times 090$ in the right eye and $+1.00 \mathrm{DS}$ in the left eye. The fundi showed pale discs with attenuated vessels and no white spots or pigmentation.

\section{Discussion}

Senior in $1961^{\prime}$ described a family where six of 13 children had nephronophthisis and tapetoretinal degeneration indistinguishable from Leber's amaurosis.

Loken in the same year ${ }^{2}$ described two siblings of non-consaguineous parents. One had mental retardation and seizures and was blind with normal appearing fundi. The second sibling was 
also blind with normal appearing fundi but was of normal intelligence. Both siblings had severe renal failure and at biopsy their kidneys showed renal tubular atrophy and dilatation. Pathological examination of the eyes showed no identifiable photoreceptors and normal retinal pigment epithelium. Nephronophthisis is an autosomal recessive condition which presents with end stage renal failure in childhood. ${ }^{3}$ Histological examination of the kidneys showed fibrosis or dilated tubules with thickened tubular basement membrane. ${ }^{4}$ There are often secondary changes in the glomeruli and cysts are present at the corticomedullary junction.

Nephronophthisis may be associated with hepatic fibrosis ${ }^{5}$ and may be familial. There are a number of ocular syndromes associated with nephronophthisis. Senior-Loken syndrome refers to nephronophthisis and retinal dystrophy. This combination in conjunction with skeletal dysplasia with cone shaped epiphyses is referred to as Saldino-Mainzer syndrome. ${ }^{6}$ Nephronophthisis, retinal dystrophy, severe deafness, diabetes mellitus, and obesity constitute Alstrom syndrome. ${ }^{7}$ Retinal dystrophy, skeletal deformities, and respiratory insufficiency are seen in Jeune's dystrophy. ${ }^{8}$ In Bardet-Biedl syndrome nephronophthisis may be present. ${ }^{9}$ Neither of the cases presented here had polydactyly or skeletal abnormalities other than mild renal osteodystrophy. Neither child was obese or diabetic.

The retinal lesion in Senior-Loken syndrome is variable and may be severe infantile onset retinal dystrophy ${ }^{11}$ or a more typical retinitis pigmentosa. ${ }^{12}$ Sector retinitis pigmentosa ${ }^{13}$ and predominant cone receptor involvement have also been reported. In addition to the features of
Senior-Loken syndrome the two cases presented here also showed moderate sensorineural hearing loss bilaterally. This has not been reported previously in association with nephronophthisis and retinal dystrophy and may represent a genetically linked condition.

These cases highlight the need for all children with a retinal dystrophy to have assessments of both their renal function and hearing. Dr Clarke is supported by the Frost Charitable Trust and the
Trent Regional Health Authority, UK, and Dr Sullivan is supported in part by the RACO/OPSM Scholarship.

1 Senior B, Friedmann AI, Braudo JL. Juvenile familial nephropathy with tapetoretinal degeneration. Am $\mathcal{f}$ Ophthalmol $1961 ; 52: 625-33$.

2 Loken AC, Hanssen O, Halvorsen S, Jolster NJ. Hereditary renal dysplasia and blindness. Acta Paediatr 1961; 50: 177-84.

3 Betts PR, Forest-Hay I. Juvenile nephronophthisis. Lancet 1973; ii: 475-8.

4 Cohen AH, Hoyer JR. Nephronophthisis: a primary tubular basement membrane defect. Lab Invest 1986; 55: 564-72.

5 Witzleben CL, Sharp AR. Nephronophthisis-congenital hepatic fibrosis. Hum Pathol 1982; 13: 728-33.

6 Mainzer F, Saldino RM, Ozonoff MB, Minagi H. Familial nephropathy associated with retinitis pigmentosa, cerebellar ataxia and skeletal abnormalities. Am 7 Med 1970; 49 $556-62$.

7 Goldstein JF, Fialkow PJ. The Alstrom syndrome: report of three cases with further delineation of clinical, pathophysiological and genetic aspects of the disorder. Medicine 1973; 52: 53-71.

8 Phillips CI, Stokes NL, Bartholomew RS. Asphyxiating thoracic dystrophy (Jeune's disease) with retinal aplasia: a sibship of two. F Pediatr Ophthalmol Strabismus 1979; 16: 279-83.

9 Hurley RM, Dery P, Nogrady MB, Drummond KN. The renal lesion of the Lawrence-Moon-Biedl syndrome. renal lesion of the Lediatr 1975; 87: 206-9.

10 Dekaban A. Hereditary syndrome of congenital retinal blind ness (Leber), polycystic kidneys, and maldevelopment of ness (Leber), polycystic kidneys, and maldeve

11 Hussels IE. Congenital amaurosis and nephrophthisis: a new syndrome. Birth Defects 1971; 7: 199-201.

12 Schimke RN. Hereditary renal-retinal dysplasia. Ann Intern Med 1969; 70: 735-44.

13 Godel V, Iaina A, Nemet $P$, Lazar $M$. Sector retinitis pigmentosa in juvenile nephronophthisis. $\mathrm{Br} \mathcal{F}$ Ophthalmo $1980 ; 64: 124-6$. 